Recherches en didactique des langues et des cultures

Les cahiers de l'Acedle

17-2 | 2020

Recherches collaboratives en didactique des langues

\title{
Oser les recherches collaboratives en DDL : transformations des chercheures, transformations des recherches
}

Emilie Lebreton et Joanna Lorilleux

\section{(2) OpenEdition \\ 1 Journals}

Édition électronique

URL : https://journals.openedition.org/rdlc/7662

DOI : $10.4000 /$ rdlc.7662

ISSN : 1958-5772

Éditeur

ACEDLE

Référence électronique

Emilie Lebreton et Joanna Lorilleux, «Oser les recherches collaboratives en DDL : transformations des chercheures, transformations des recherches », Recherches en didactique des langues et des cultures [En ligne], 17-2 | 2020, mis en ligne le 27 avril 2020, consulté le 17 octobre 2022. URL : http:// journals.openedition.org/rdlc/7662 ; DOI : https://doi.org/10.4000/rdlc.7662

Ce document a été généré automatiquement le 17 octobre 2022.

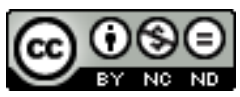

Creative Commons - Attribution - Pas d'Utilisation Commerciale - Pas de Modification 4.0 International - CC BY-NC-ND 4.0

https://creativecommons.org/licenses/by-nc-nd/4.0/ 


\title{
Oser les recherches collaboratives en DDL : transformations des chercheures, transformations des recherches
}

\author{
Emilie Lebreton et Joanna Lorilleux
}

1 Les finalités des recherches collaboratives, souvent rapportées à la compréhension et à l'amélioration des pratiques, en mobilisant des points de vue de professionnels, donnent lieu à des réflexions qui s'articulent essentiellement sur les procédés, les actions réalisées et les perspectives à tirer en termes de recherche et de formation.

2 Nous chercherons à mettre en lumière, par une mise en discussion réflexive, les enjeux éthiques et méthodologiques liés à nos choix épistémologiques. Nous réinterrogerons ainsi au prisme de nos expériences des catégories qui semblaient pourtant, de prime abord, évidentes (praticiens/chercheurs, expertise/engagement par exemple). Comment une épistémologie, plaçant en amont de toute compréhension l'expérience $\mathrm{du}$ chercheur (histoire, projets et conceptualité au sein desquels s'opère la compréhension) et la relation aux autres vient-elle potentiellement récuser l'existence de frontières fixes entre ces catégories?

3 Cet article, écrit à quatre mains, est un travail collaboratif entre deux chercheures, et il nous a semblé important d'y faire entendre, par moments, la pluralité de nos voix.

\section{Saisir le flou?}

\section{Traits définitoires}

4 Les recherches collaboratives ont la particularité d'offrir la possibilité de chercher autrement. Présentées comme une démarche de recherche originale alliant la construction d'actions à la production de savoirs, les recherches collaboratives en didactique des langues englobent toutes formes de recherche rapprochant le monde 
académique et celui des professionnels autour de thématiques et de préoccupations communes. Si ces premières caractéristiques les distinguent des recherches dites théoriques ou appliquées - où l'accent est mis sur la conceptualisation, et où le va-etvient entre l'action et la recherche est moindre - il convient d'ajouter que ce sont les dimensions interventionnistes et pragmatiques qui font que ces recherches s'imposent dans le domaine de la didactique des langues, et plus largement dans la recherche en éducation et formation. Des éléments de définition fournis par différents travaux ${ }^{1}$ plaçant les recherches collaboratives au centre de leur réflexion, nous retenons que cette "méthodologie d'intervention" (Morissette, $2013: 41$ ) a pour objectif premier de produire des savoirs visant à faire évoluer les pratiques, à pallier les problèmes et difficultés repérés. Pour y parvenir, la collaboration avec des professionnels, tant pour comprendre leurs pratiques que pour les améliorer, représente un procédé pertinent et judicieux où savoirs théoriques et d'expériences se côtoient, se confrontent, voire se (re)construisent. Toutefois, travailler avec ne semble pas totalement suffisant, il est également nécessaire de tenir compte des finalités et des intérêts respectifs pour maintenir l'esprit collaboratif visé, notamment en favorisant cet échange de services permettant le développement professionnel des enseignants et la production de connaissances scientifiques dans un champ précis (Desgagné et al : 2001).

5 En condensant ainsi les caractéristiques de ces recherches, nous soulignons leurs aspects utiles, applicables et stimulants, sans aborder les subtilités qui font, en partie, des recherches collaboratives un sujet à discussion. Ces points de discussions concernent à la fois les finalités et les modalités de ces recherches: il est autant possible de s'inscrire dans une dynamique d'intervention sur le réel par la transformation des pratiques (Hugon \& Seibel, 1988) que de privilégier une conscientisation des pratiques sans chercher à les transformer (Montagne-Macaire, 2007). Le jeu de relations qui se crée est aussi évoqué : il peut s'agir d'accorder un statut égal aux chercheurs et enseignants dans la définition de l'objet de recherche et la production de savoirs (Morissette, 2013. Desgagné, 1997) ou d'aller plus loin en intégrant les points de vue des praticiens dans la construction des connaissances. De nombreuses explicitations visent à faire plus clairement la distinction entre les diverses appellations existantes (recherche collaborative, recherche-action, rechercheintervention, recherche-action collaborative, etc.). Elles ont leur importance pour donner une direction au projet de recherche, mais nous nous concentrerons ici sur ce qui semble constitutif de cette forme de recherche, à savoir une réflexion, passant par un croisement des regards, sur les pratiques, et exclusivement sur les pratiques (de professionnels).

\section{Des pratiques, de bout en bout}

6 Les pratiques sont emblématiques des recherches collaboratives. Peu importe le stade de recherche auquel on se trouve et l'objectif affiché interroger, remettre en question, agir, construire 㡠 les pratiques demeurent «l'objet d'exploration» (Desgagné et al., 2001 : 37) autour duquel chercheurs et professionnels travaillent, ensemble. L'accent étant principalement mis sur elles, les pratiques forment une évidence sur laquelle on ne s'arrête plus. Ces pratiques sont pourtant à l'origine des recherches impulsées par des praticiens, des chercheurs, ou des institutions. Elles constituent un socle où s'ancrent :

les relations entre professionnels et chercheurs, les intérêts et les rôles de chacun ; 
les savoirs valorisés, à construire ;

la manière de concevoir et de faire de la recherche collaborative ;

la possibilité même de s'inscrire dans une recherche collaborative, les rencontres, les finalités.

Les deux premiers points mentionnés font l'objet de réflexions, mais toujours au prisme des pratiques.

En effet, dans l'optique de rétablir un rapport entre deux univers professionnels, les travaux mobilisant les notions de recherche collaborative, recherche-action ou recherche-intervention, veillent à discuter des relations par le biais des modalités de travail où l'enseignant n'est pas vu comme un simple acteur pourvu d'une indispensable compétence justifiant sa participation :

«La recherche collaborative s'inscrit, en ce sens, dans le mouvement de substitution de l'image mécaniste de "l'enseignant efficace", conçu comme le « docile exécutant » des prescriptions du chercheur, vers celle, plus constructiviste, $\mathrm{du}$ « praticien réflexif » conçu comme le «partenaire averti » qui contribue, avec le chercheur, dans une réflexivité conjointe, au développement de la pratique » (Desgagné et al., $2001: 35$ ).

Nombre de chercheurs s'engageant dans des recherches collaboratives prennent position pour souligner la contribution essentielle des professionnels, de leurs regards, de leur rôle pour faire évoluer les questions de recherche. Cette prise de position apparait par ailleurs dans des appels à projets ${ }^{2}$ émanant de diverses institutions et dans lesquels on peut lire l'intérêt de valoriser les savoirs d'expériences, notamment pour faire avancer les connaissances.

10 Néanmoins, il apparait très souvent que ces recherches permettent également de favoriser le développement d'une réflexion des professionnels sur leurs pratiques, encourageant les enseignants à les remettre en cause. Cela permet de redonner un pouvoir d'agir aux formateurs (Morrissette, 2013 ; Desgagné, 1997). L'accompagnement des chercheurs soulève des questions qui vont au-delà des simples modalités et procédés. Comment s'assurer que les chercheurs «éclairent » les professionnels ? Ou que ces derniers attendent d'être "éclairés » par les chercheurs? Les professionnels ont-ils besoin de faire de la recherche pour interroger leurs pratiques? En maintenant une logique de « lumière » apportée par le monde de la recherche à celui de la pratique, et en ne mettant pas autant l'accent sur l'apport des professionnels au développement professionnel des chercheurs, on peut se demander s'il n'y a pas un risque de réduire et de circonscrire ce qui s'opère lors de ces collaborations.

11 Y. Bonny propose une typologie, élaborée en fonction de la place accordée à chacun des acteurs (chercheur/praticien) et des relations qu'ils entretiennent autour de l'élaboration des savoirs issus de ces recherches. La typologie procède par crescendo, depuis les configurations où le travail de recherche est réservé aux chercheurs, jusqu'aux configurations où savoirs théoriques et praxéologiques sont considérés à parité. Quelle que soit la configuration envisagée, Bonny souligne l'importance de "penser [...] la pertinence praxique de la connaissance produite, ou plus fortement le déploiement de l'activité de recherche comme composante d'une praxis » (2015:5).

Il nous semble que le prisme des pratiques contribue à une forme de hiérarchisation des recherches, qui iraient à rebours du crescendo mentionné plus haut: dans la mesure où la conceptualisation tend à ne plus être centrale, l'intervention et les savoirs produits sont, le plus souvent, réduits à un résultat qui doit être visible, concret, 
applicable. Finalement, de même qu'une trop forte centration sur le "référentiel scientifique » (ibid.), la seule préoccupation pratique risque à son tour de limiter la collaboration à un «milieu de pratiques " pour les uns et un «terrain d'enquête " pour les autres (la théorie se construisant au mieux en aval de la collaboration). Cela pourrait avoir pour conséquence de ne laisser place qu'à un type de relations pas aussi équilibrées qu'on aurait pu l'imaginer.

Face à la multiplicité des critères et des appellations visant à désigner les recherches dites collaboratives, dubitatives ${ }^{3}$ quant à l'intérêt des éléments de taxinomie évoqués en première partie, nous souhaitons poursuivre la réflexion à partir de recherches que nous avons menées pour en explorer les dimensions fondamentales, à savoir éthiques et épistémologiques.

\section{Accentuer le flou}

14 Nous nous appuyons ici sur plusieurs de nos expériences de recherche (thèses, dispositif élaboré autour des questions de langues et insertions), toutes ancrées dans un paradigme qualitatif et interprétatif, où l'importance des autres participe pleinement de toute compréhension (Lorilleux, 2015 ; Lebreton, 2017). Nous en présentons les éléments qui nous semblent pertinents pour la discussion, en mettant en évidence les limites que nous voyons à l'élan taxinomique et en soulignant la centralité, dans ces expériences, de la rencontre altéritaire.

Notre écriture commune ne peut laisser se confondre nos voix, nos regards, nos expériences. C'est pourquoi nous avons fait le choix d'utiliser l'italique, précédé des initiales de l'énonciatrice (E.L. pour Emilie Lebreton et J.L. pour Joanna Lorilleux) pour marquer les passages où le croisement, le frottement de nos expériences fait sens. Les passages sans italiques correspondent à des compréhensions partagées des phénomènes présentés.

\section{Praticiens, chercheurs : tous des professionnels}

JL: Professeure des écoles auprès d'élèves allophones nouvellement arrivés, j'ai été mise en recherche par la rencontre d'élèves auxquels mon savoir-faire pédagogique, j'en avais le sentiment, n'était pas adapté. Face à ce sentiment, j'ai cherché à comprendre ce qui, au-delà des techniques pédagogiques mises en cuvre, pouvait jouer dans ce qui m'apparaissait comme une non appropriation de la lecture et de l'écriture en français. Ma mise en recherche trouve sa source dans des situations de classes jugées inopérantes par la praticienne que j'étais, mais aussi dans l'expérience partagée avec ces élèves, qui me permettait de comprendre que le problème ne se limitait pas à une question de technique pédagogique. A cette première rencontre s'ajoute une autre expérience d'abord déstabilisante : celle de l'animation de formations d'enseignants où les alternatives présentées ne trouvent pas d'écho favorable chez les participants. Cela m'a poussée à proposer à l'une des collègues présentes de mettre en place, au sein de sa classe, des séances d'arts plastiques, comme chemin détourné vers l'appropriation de l'écriture en français. Mise en place que j'ai également proposée à une enseignante du réseau FLS d'Indre-et-Loire au sein de sa classe NSA de collège. Ces propositions ont été concomitantes avec mon inscription en thèse de doctorat et ont servi de base à ma recherche.

Cette recherche de thèse part donc de besoins identifiés par des professionnels :

- praticienne mise en recherche par ses élèves ; 
- formatrice collaborant avec une enseignante pour remédier à une situation de classe jugée critique ;

- collègues enseignantes - dont une apprentie-chercheure - proposant des activités moins scolaires à des élèves non scolarisés antérieurement, pour varier les approches de classe. observations formulées précédemment (1.2). À l'instar de plusieurs chercheurs en DDL la mise en recherche s'est réalisée au cours d'une expérience d'enseignement. Plus précisément, il s'agissait pour Joanna selon les termes d'Y. Bonny "d'être en recherche » au sens d'être confrontée à une situation insatisfaisante. Par la suite, sa "mise en recherche" s'est réalisée de diverses manières: en s'impliquant dans une formation et dans la mise en place d'une forme de recherche-action qui s'est développée en "faisant de la recherche", par l'élaboration d'une étude doctorale discutée et reconnue par la communauté scientifique, " respectant les canons en vigueur du référentiel scientifique, dont les chercheurs professionnels sont les experts et les garants" (Bonny, 2015: 2). Plus que de simples étapes correspondant à des formes précises de recherches collaboratives, ces distinctions rappellent que les praticiens peuvent déjà être dans une démarche de réflexion et de recherche sans nécessairement être impliqués dans un dispositif formel de recherche. Mettre en évidence ce type d'implicite influe sur la manière de concevoir la recherche et de la faire. En outre, la mise en recherche, évoquée plus haut, résulte de rencontres, de collaborations avec d'autres personnes: apprenants, enseignants, experts, amis à l'occasion. Le fait de le mentionner, et de le souligner ici, permet de rappeler qu'en dehors des recherches collaboratives "officielles", institutionnalisées, il se passe des choses ${ }^{4}$ qui ne sont pas nécessairement inscrites dans le carcan du "référentiel scientifique ».

ettre en regard nos expériences nous permet de déplacer le projecteur sur des acteurs qui demeurent parfois au second plan ou maintenus dans un rôle de praticien, et de souligner qu'à travers leurs expériences, réceptions et réactions, ils participent au développement des recherches et transforment nos pratiques professionnelles de chercheurs.

23

EL: Soucieuse d'intégrer le point de vue des autres et d'articuler la réflexion à l'action, j'ai souhaité travailler étroitement avec des professionnels et des apprenants pour bousculer leurs regards et mettre en mouvement mes propres questionnements. Si le projet de thèse auquel je répondais visait à optimiser les pratiques de formation, j'ai préféré réfléchir avec les acteurs de la recherche à des propositions didactiques alternatives portant sur les manières d'être dans les situations d'enseignement-apprentissage et leurs répercussions sur les manières d'agir. J'ai alors 
déployé une méthodologie plurielle offrant la possibilité de mobiliser les savoirs d'expériences des professionnels, d'analyser leurs pratiques en s'y confrontant, $y$ compris à partir des observables construits avec les apprenants. Les pratiques ont constitué un tremplin, un prétexte ou encore un élément déclencheur à nos réflexions. Des rencontres ont également été provoquées par l'organisation et notre participation conjointe à des ateliers interprofessionnels, tant sur la rive droite du fleuve où se trouve l'université, que sur la rive gauche où les centres de ressources et de formation sont majoritairement implantés. Pour ma part, il s'agissait de faire se rencontrer les acteurs; de favoriser un espace d'échanges; de créer un réseau de travail et de réflexion. Au-delà d'une réflexion enrichie par croisements de regards et d'expériences, cette manière de chercher est agréablement inconfortable. Ainsi, lors d'ateliers pédagogiques organisés par des professionnels pour leurs pairs, les vives réactions engendrées par les présentations des chercheures et les discussions qui ont suivi sur l'absence de réponses immédiates, d'outils clés en main et des prescriptions "farfelues et déconnectées de la réalité", sont de précieux éléments pour réfléchir au sens de nos actions.

24 JL: L'expérience présentée ici par Emilie a tous les atours d'une recherche collaborative: des praticiens sollicitent des chercheurs, la Région finance une thèse dans la perspective de mobiliser une diversité d'acteurs (de "terrain» - praticiens - et "hors sol " - chercheurs-) dans le but d'améliorer son action formative envers les migrants. La chercheure y fait l'expérience inconfortable d'une forme d'incommunicabilité. L'attention portée à la réception, par les acteurs de terrain, des propositions finalement énoncées par des universitaires semble mettre en évidence des éléments parfois tus sur les recherches collaboratives: les relations entre les participants à la recherche sont pour beaucoup dans la réussite des projets, dans la pérennité et l'appropriation des connaissances construites à travers ces recherches. Cet inconfort relatif est pourtant mis à profit par la chercheure qui choisit de s'engager dans une réflexion sur ce qui lui permettrait de "tenir " (Gaspar, 2012) les deux bouts, voire d'en attraper un autre.

Ces deux premiers exemples nous permettent a) d'illustrer les limites de la classification définitoire des recherches collaboratives et b) de percevoir les limites de la réception de certaines de ces recherches dites "collaboratives", qui ne parviennent pas (toujours) à produire des résultats validés par les deux parties engagées: " praticiens » et " chercheurs 》.

Loin de nous décourager, ces éléments de déconstruction des représentations sur les recherches collaboratives nous ont poussées à nous engager ensemble sur d'autres voies, attachées que nous sommes aux deux rives de ces recherches: la pratique et la réflexion théorique, en quête, à notre tour, d' " une autre manière de chercher " (Mesnier \& Missotte, 2003).

\section{Diversifier les recherches collaboratives}

27 Pour réfléchir aux enjeux méthodologiques et épistémologiques des recherches collaboratives, nous avons choisi de mobiliser deux autres expériences dont nous sommes à l'origine, avec d'autres chercheurs ${ }^{5}$. Nous avons sollicité des formes de collaboration que nous allons décrire rapidement, de manière à y souligner les éléments pertinents pour notre réflexion.

\section{Le QUILT, un site internet à l'attention de formateurs (bénévoles notamment) intervenant dans le champ de la formation linguistique pour migrants}

28 A l'occasion de la «crise " migratoire de 2015, de nombreuses images de bénévoles proposant des cours de français aux réfugiés ont fait la une des médias. Certains reportages illustrant la bonne volonté de bénévoles non formés ont renforcé notre envie de nous mobiliser en mettant à profit notre spécificité d'universitaires œuvrant 
dans le domaine du français en contexte migratoire. Nous avons choisi de nous lancer dans l'élaboration d'un site internet proposant une ouverture réflexive aux personnes qui nous liraient. Les dimensions collaboratives de cette expérience résident dans le fait que nous avons souhaité soumettre notre travail à des partenaires issus du milieu associatif', afin d'améliorer la réception de notre site auprès du public auquel nous avions pensé l'adresser. Ce travail procède d'un va-et-vient entre :

- des savoirs construits avec des praticiens et des «usagers" des cours de français (nous avons utilisé pour concevoir les contenus du site des questionnements et des savoirs issus entre autres 區 de nos thèses) et

- la réception de ces éléments par d'autres praticiens, réception produisant elle-même des effets sur le positionnement et la présentation de ces savoirs.

C'est en quelque sorte un mouvement de retournement de la demande habituellement formulée dans les recherches collaboratives, dans la mesure où nous, universitaires, avons souhaité échanger avec des praticiens pour améliorer notre pratique de professionnels de la recherche (sur le plan notamment de la diffusion des connaissances).

30 Cette collaboration avec des praticiens trouve une forme de prolongement dans un autre dispositif pensé dans un même élan de collaboration, mais sans lien direct avec le QUILT : les ateliers interprofessionnels.

\section{Des ateliers interprofessionnels à l'université ?}

31 Comme le QUILT, ces ateliers procèdent de notre volonté de croiser des regards de praticiens et de chercheurs sur des questions communes dans le cadre de la formation linguistique des migrants, pour affiner les compréhensions - par chacun [50[20 de ces phénomènes. Nous nous sommes saisies de l'occasion d'un colloque ${ }^{7}$ organisé par notre équipe de recherche pour inviter des professionnels, des bénévoles et des usagers ${ }^{8} \mathrm{du}$ FLE/S ainsi que les participants au colloque à venir échanger sur la notion d'insécurité linguistique. Ce colloque a constitué le premier jalon d'un cycle d'ateliers qui se poursuit, autour de notions clés du champ de la formation linguistique pour migrants (in/sécurité linguistique ; langues - insertions - terrains d'intervention ; engagement ; diversité). Le succès rencontré par ces ateliers fait naitre une dynamique d'échanges propice à alimenter nos réflexions théoriques sur le domaine, mais aussi sur le rôle et la place que nous occupons, comme chercheures, dans la société.

Ces deux expériences mobilisent de façon croisée les savoirs dits savants et d'expérience autour d'un champ de recherche; y participent des praticiens, des usagers (minoritairement, toutefois à ce stade) et des chercheurs. Ces croisements stimulent la réflexivité des uns et des autres qui se laissent interroger, déplacer par les altérités perçues. L'altero-réflexivité ${ }^{9}$ (Robillard, 2007 ; Bretegnier, 2009 ; Feussi, 2010, 2018) à l'œuvre apporte au caractère collaboratif qui influe ainsi sur les pratiques ... de recherche.

33 A la différence de ce qui est d'usage dans les recherches collaboratives, ce sont ici les chercheures qui ont sollicité l'expertise (ou les savoirs) des "praticiens", en leur soumettant tantôt les contenus élaborés dans le QUILT, tantôt des concepts construits par des chercheurs, et ce dans l'optique de se confronter à la réception de personnes occupant d'autres «fonctions ", d'autres rôles, et de tenir compte de leurs retours pour faire évoluer, ensemble, ces projets. Du reste, les rencontres proposées se déroulent sur 
l'espace professionnel des chercheures, et les modalités de travail collaboratif sont également plus proches de celles de l'université que de celles des centres de formation, dans la mesure où la parole y circule à partir de supports semblables à ceux mobilisés lors des séminaires de recherche de l'équipe. Ces recherches ne se développent pas en toute «symétrie » entre les différents acteurs, mais les idées et la réflexion prennent sens et forme au gré des rencontres et discussions.

Ces deux expériences se sont révélées déstabilisantes pour les deux parties. Pour certains professionnels, notre choix de ne pas nous situer dans une dynamique de "demande-réponse ", pour éviter de coller au plus près d'aspirations utilitaires, peut générer de l'incompréhension voire de l'agacement. Pour les chercheures, s'autoriser à procéder ainsi suscite parfois des doutes liés à la validation et à la légitimité de recherches qui se veulent "collaboratives", sans protocole explicite et sans expérimentation sur le terrain. Nous poursuivons pourtant, en saisissant notre inconfort, mais un «inconfort positif!» évoqué par J.-P Astolfi (1993 : 17), et en assumant de potentiellement construire des savoirs «incertains, qui deviennent le moteur de la réflexion sur l'action » (Morrissette, $2013: 47$ ), l'action incluant aussi pour nous la théorisation. Plus que des résultats, les fruits de nos démarches prennent la forme d'une "instabilisation réflexive occasionnée par les frottements altéritaires " (Castellotti, Debono \& Huver, 2017 : 72)

\section{A la recherche des résultats perdus?}

\section{Coénoncer des connaissances spécifiquement « métissées » ?}

L'ensemble de nos recherches - rapidement présentées ici - s'inscrit dans le paradigme herméneutique décrit par Astolfi, dans le sens où celles-ci sont centrées "sur l'interprétation et la recherche de significations » (1993:6); mais elles relèvent selon les cas de l'une ou l'autre "coupe » qu'il opère: propositions d'alternatives (qu'il nomme innovations sans adhérer nécessairement au caractère innovant des propositions pédagogiques mentionnées) ; puis interprétations des pratiques, autour de ce qu'il nomme à la suite de M. Bataille " un chercheur collectif» (1993:16). Si nous avons pu nous reconnaitre dans de nombreux traits mobilisés par Astolfi pour comprendre les recherches participatives, nous devons mettre en question cette idée de "chercheur collectif», qui semble trouver écho dans d'autres écrits, amenant parfois les chercheurs à viser (et/ou revendiquer) une forme de co-énonciation du sens fait de ces recherches. Des connaissances, « des savoirs, le plus souvent coénoncés et covalidés » (Bourrassa \& Boudjaoui, $2012: 5$; cités dans l'appel auquel nous répondons) dont la nature est parfois vue comme "métissée [...] » dans la mesure où les frontières entre savoirs expérientiels et savoirs savants sont justement un espace de coconstruction des significations (Miguel Addisu \& Maire-Sandoz, 2018)» (citées dans le même appel). Ces derniers éléments nous semblent intéressants à discuter, en déplaçant leur conceptualisation vers une orientation phénoménologiqueherméneutique, reposant sur l'idée selon laquelle toute compréhension est toujoursdéjà expérientielle, avant, ou en même temps que rationnelle, et par conséquent, toujours singulière, parce que se détachant d'un fond antéprédicatif (Romano, 2010; Castellotti, et al., 2017). 
$\mathrm{Si}$, comme nous, l'on admet cette diversité des interprétations comme indépassable, parce que toujours inscrite dans une histoire singulière, orientée vers un projet particulier, et s'élaborant dans une conceptualité propre, cela interdit - en théorie - la possibilité d'une co-énonciation de savoirs. En effet, dans les exemples que nous avons mobilisés, nous repérons aisément des éléments qui échappent aux différents participants : les «praticiens », les « chercheurs » ou les « usagers ${ }^{10}$ » s'approprient les concepts travaillés en les nouant à leur propre expérience, en les orientant vers leurs propres projets de compréhension. Ceci nous amène à penser que toute (nouvelle) connaissance est toujours-déjà métissée: toute connaissance, s'inscrivant dans la corporéité, dans l'imaginaire, dans l'histoire, dans les visées de celui qui l'énonce, comme de celui qui la reçoit est nécessairement en partie expérientielle :

«Dès lors, le sens est à concevoir non seulement comme pluriel car expérientiel, perçu différemment par chacun, mais aussi comme pétri d'altérité et, de ce point de vue, au moins partiellement «inaccessible » (...). Plus exactement, cette altérité fondamentale suppose des formes de traduction, qui ne peuvent pas passer par des procédures technicisées et reproductibles (i.e. des outils, des protocoles, des démarches) mais par l'instauration d'une relation et d'un projet ( $\mathrm{au}$ moins partiellement partagé). La réflexivité occupe par conséquent une position centrale, « en tant que processus de constitution du sens des autres solidairement par transformation à partir du nôtre (...), [pour] susciter des conflits productifs en cela que nos propres repères implicites sont instabilisés et visibilisés si nous parvenons à imaginer que d'autres créent du sens pour être de manière très différentes de la nôtre jusqu'alors » (Robillard, $2013: 53$ )» (Castellotti, et al, $2017: 69$ ).

La question des protocoles de recherche, des dispositifs collaboratifs se voit ici déplacée sur le plan de la relation entre les participants, qui doivent être en mesure d'instaurer un projet « au moins partiellement partagé ».

Cette perspective amène une autre question au moins: est-il possible de décréter l'instauration d'une relation et d'un projet commun dans un cadre institutionnel qui pousse de plus en plus le monde académique à collaborer avec des "professionnels » pour répondre à des besoins exprimés dans une " demande »? Autrement dit, peut-on (ou suffit-il de) penser un dispositif collaboratif au sein duquel « on » ferait travailler ensemble des praticiens forts de leurs savoirs d'expérience et des chercheurs forts de leurs savoirs théoriques et savoir-faire, autour d'un problème identifié en amont, pour parvenir à produire des connaissances nouvelles « coénoncées » par les participants à la recherche?

Nous avons montré plus haut que la distinction entre praticiens, chercheurs, et usagers n'est que partiellement pertinente, nous pourrions souligner à présent cette évidence : l'altérité n'a pas nécessairement besoin de statuts professionnels différents pour se faire jour. Praticiens, chercheurs, usagers, une même personne peut avoir fait l'expérience de ces trois statuts, et en viendra donc probablement à mobiliser des connaissances issues de toutes ces expériences pour construire de nouvelles connaissances. Attention, il ne s'agit pas pour autant d'homogénéiser ces statuts, mais bien de reconnaitre une fluidité, une perméabilité et de souligner le caractère mouvant des « connaissances » produites dans le cadre de ce type de recherches. Cette diversité des statuts, des expériences, mène à des significations toujours nécessairement diverses elles aussi, du fait même de leur inscription dans les expériences des participants. vraiment parvenir à une co-énonciation des savoirs. Elles peuvent «co-construire des 
significations ", à condition que l'on admette que ces significations ne sont jamais tout à fait les mêmes pour chacun des participants, qui peuvent s'accorder plus ou moins implicitement - dans une disposition ouverte à l'autre - sur une forme de consensus fictionnel. En partie fictionnel, certes, mais indispensable pour parvenir à la transformation de chacun. Finalement plutôt que de chercher à "co-construire " des connaissances, ce qui risquerait de mener à une imposition à l'autre (si minime soitelle) du sens construit par l'un, nous optons pour une forme de collaboration qui assume la possibilité d'une transformation réciproque.

\section{Conditions de possibilité de transformation(s) réciproque(s)}

Cette réciprocité transformative re-pose à son tour la question de la relation à instaurer (cf. Castellotti, et al. 2017 supra) dans le cadre institutionnel de "la recherche » : pour se laisser transformer par la rencontre altéritaire (qui pourrait être considérée comme le point nodal des recherches collaboratives), il faut que les participants se trouvent dans une certaine disposition. Cette disposition ne saurait être réduite au projet commun d'élucider une question de recherche, plus ou moins codécidée» (Bourrassa \& Boudjaoui, 2012:5) : l'on trouverait dans les exemples que nous avons mobilisés plus haut des personnes avec qui nous n'avons pas pu travailler, nous trouvant dans une relation stérile. Ce qui rend possible une transformation réciproque au sein de recherches collaboratives repose sur le fait (pas toujours, voire rarement explicité) d'être concerné, affecté par le travail collaboratif qui s'ouvre et s'opère. La relation qui s'instaure (dans le meilleur des cas) s'instaure dans une «tonalité affective", une "Stimmung " qui traverse chacun sans que l'intentionnalité y soit impliquée. Cette tonalité affective «nous présente [...] le monde comme ce qui nous concerne, nous affecte et donc appelle à la compréhension" (Camilleri, 2008: 201). Pour le dire plus prosaïquement, on pourrait parler d'une « longueur d'onde », ou d'une coloration, au sein de laquelle nous sommes au monde et qui fait partie de la conceptualité (rapidement mentionnée plus haut) au sein de laquelle advient le sens que nous faisons des phénomènes. Cette tonalité affective, que nous pouvons parfois ressentir, nous échappe en même temps, ne se laisse pas contrôler. Nous ne pouvons ici mobiliser que très superficiellement cette notion, mais elle nous permet de souligner les dimensions antéprédicatives dont il nous semble important de ne pas faire l'économie quand il s'agit de réfléchir aux dimensions épistémologiques des recherches collaboratives, en sciences humaines.

\section{Assumer une part (d')incontrôlable}

Ecrire ce texte nous a permis de réfléchir à notre façon de faire de la recherche, en collaborant avec d'autres. Nous avons procédé à un tour d'horizon (non exhaustif) des travaux sur la question, que nous avons confrontés à nos propres expériences de recherches. Cela nous a amenées à questionner le sens des catégories mobilisées au sein $\mathrm{du}$ paradigme collaboratif (recherche action, recherche intervention, recherche participative, praticiens, chercheurs, théorie, pratique, savoirs d'expérience et savoirs théoriques, etc.). Nous avons aussi souhaité nous interroger sur la question de la coconstruction de savoirs partagés, ce qui nous a amenées à expliciter des orientations épistémologiques qui remettent en question l'universalité du sens, et son extériorité, déplaçant ainsi la centration sur les résultats vers une attention particulière portée à la 
relation entre les participants, à la nature des savoirs issus de ce type de recherches, et aux dimensions antéprédicatives qui les innervent. Autant de réflexions qui nous amènent à présent à nous interroger de nouveau : qu'est-ce qui nous permet de nous positionner face au paradigme des recherches collaboratives? Des protocoles de recherche? Les méthodologies déployées? Sans doute non, ceux-ci étant en partie invalidés par l'importance que nous avons souhaité accorder aux dimensions antéprédicatives : les relations humaines, la disposition à se laisser transformer lors de rencontres altéritaires, «ça ne se commande pas». Alors quoi? Au risque de formuler une tautologie, disons qu'une recherche collaborative, c'est une recherche qui se fait avec des autres, qui porte en soi une hétérogénéité de compréhensions, et que l'on ne peut viser à réduire cette hétérogénéité dans l'homogénéisation du sens.

\section{BIBLIOGRAPHIE}

Astolfi, J.-P. (1993). « Trois paradigmes pour les recherches en didactique », Revue française de pédagogie, 103, 5-18.

Bonny, Y. (2015). « Les recherches partenariales participatives : Ce que chercher veut dire », dans Lyet, P. (dir.), Les recherches-actions collaboratives : Une révolution de la connaissance, Rennes : Presses de l'EHESP, 1-6.

Bourrassa, B \& Boudjaoui, M. (dir.) (2012). Des recherches collaboratives en sciences humaines et sociales : enjeux, modalités et limites. Québec : Presses de l'Université Laval.

Bretegnier, A. (2009). « Sociolinguistique alter réflexive : Du rapport au terrain à la posture du chercheur ». Cahiers de sociolinguistique, 14(1), 27-42.

Camilleri, S. (2008). « Tonalité affective (Stimmung) ». Dans Beaulieu, A., Abécédaire de Martin Heidegger, Mons : Sils Maria éditions, 200-202

Castellotti, V., Debono, M., Huver, E. (2017). « D’une didactique contextualisée à une didactique diversitaire ». Dans Babault, S., Bento, M., Spaëth, V., Tensions en didactique des langues. Entre enjeu global et enjeux locaux, Bruxelles : P.I.E.-Peter Lang, 49-76.

Desgagné, D. (1997). « Le concept de recherche collaborative : l'idée d'un rapprochement entre chercheurs universitaires et praticiens enseignants ", Revue des sciences de l'éducation, 23 (2), 371-393.

Desgagné, S., Bednarz, N., Lebuis, P., Poirier, L. \& Couture, C. (2001). « L'approche collaborative de recherche en éducation : un rapport nouveau à établir entre recherche et formation ». Revue des sciences de l'éducation, 27 (1), 33-64.

Feussi, V. (2010) «Usages linguistiques et constructions identitaires au Cameroun. A la recherche de soi et/avec l'autre?», Cahiers de sociolinguistique, 15 (1), 13-28.

Feussi, V. (2018) Francophonies - relations - appropriations Une approche historicisée et expérientielle des « langues ». Note de synthèse HDR, soutenue le 9 novembre 2018 à l'Université de Cergy-Pontoise. 
Gaspar J.-F. (2012). Tenir! Les raisons d'être des travailleurs sociaux. Paris : La Découverte.

Guyon, R. (2018). Editorial, Diversité, 192-6.

Hugon, M.-A., \& Seibel, C. (1988). Recherches impliquées recherches-actions : le cas de l'éducation, Bruxelles : De Boeck-Wesmael.

Lebreton, E. (2017). Pour des formations linguistiques (trans)formatrices : renverser les évidences pour penser l'appropriation du français par des adultes migrants, Thèse de doctorat soutenue le 11 septembre 2017 à l'université de Rouen.

Lorilleux, J. (2015). Ecritures transformatives. Quand des élèves allophones deviennent auteurs, ou : de l'appropriation à l'émancipation, Thèse de doctorat soutenue le 5 décembre 2015 à l'université François Rabelais de Tours.

Montagne-Macaire, D. (2007) « Didactique des langues et recherche-action », Recherches en didactique des langues et des cultures [En ligne], 4 | 2007, mis en ligne le 21 août 2007, consulté le 02 mars 2020. URL : http://journals.openedition.org/rdlc/5071.

Mesnier, P.-M., \& Missotte, P. (dirs.) (2003) La recherche-action, une autre manière de chercher, se former, transformer, Paris : L'Harmattan.

Miguel-Addisu, V. \& Maire-Sandoz, M.-O. (2018). « Enseigner la lecture dans une classe plurilingue et défavorisée ", dans : Briquet S. (dir.) Enseignement-apprentissage de la lecture, Carrefour de l'Éducation $n^{\circ} 46,15-26$.

Morrissette, J. (2013). « Recherche-action et recherche collaborative : quel rapport aux savoirs et à la production de savoirs? », Nouvelles pratiques sociales, 25(2), 35-49.

Robillard, D. de (2007) «La linguistique autrement : altérité, expérienciation, réflexivité, constructivisme : en attendant que le Titanic ne coule pas ", CAS, 50 ans après le Cours de Saussure : la linguistique en question, 1, Université de Picardie, 81-228.

Robillard, D. de (2013). « Réflexivités, qualitativités, ou humanités? ». Texte présenté lors des Tours Qualitatifs 2013, Université F. Rabelais (Tours), EA 4246 PREFics-DYNADIV, 27 juin 2013.

Romano, C. (2010). Au cœur de la raison, la phénoménologie, Paris : Gallimard.

Serres, M. (1980). Le passage du Nord-Ouest, Paris : Minuit.

\section{NOTES}

1. Nous faisons principalement référence aux travaux de chercheurs francophones souhaitant conceptualiser les recherches collaboratives, notamment en interrogeant leurs enjeux, limites et perspectives.

2. A titre d'exemples: https://www.culture.gouv.fr/Sites-thematiques/Langue-francaise-etlangues-de-France/Politiques-de-la-langue/Langues-et-numerique/Appel-a-projets-nationalLangues-et-numerique-2017 (consulté le 12/02/2020) et http://ife.ens-lyon.fr/lea/le-reseau/ @@carte_des_leas (consulté le 12/02/2020). Voir également à ce propos l'Editorial de R. Guyon (2018) et certains articles qui composent le ${ }^{\circ} 192$ de la revue Diversité.

3. Ces typologies démultipliées risquent d'attirer l'attention des personnes qui les mobilisent sur une forme de foisonnement d'appellations qui pourraient bien y perdre leur sens. Il nous semble en effet plus fondamental d'en explorer les dimensions éthiques et épistémologiques que de bien choisir l'étiquette (quitte à en créer de nouvelles au moindre frémissement de différence) qui servira à se distinguer d'autres recherches. Ce mouvement nous amène peut-être à un manque de précision, mais nous choisissons d'en assumer le flou. 
4. Nous empruntons à J.-P. Astolfi (1993: 9) cette belle citation de M. Serres « Combien de pêcheurs basques, à la course à la baleine, combien de Norvégiens, de Grecs, de Phéniciens, de Bretons inconnus, avaient découvert l'Amérique et le trou vers le Pacifique, avant le savant érudit Christophe Colomb, représentant des Rois ? Ils ne l'ont pas écrit, voilà toute l'affaire » (Serres, 1980 :60).

5. Le QUILT est un projet de l'équipe DYNADIV (EA4428, Université de Tours), auquel collaborent centralement L. Courtaud, E. Lebreton, J. Lorilleux et C. Rubio, et qui a été initié sous l'impulsion de V. Castellotti et E. Huver. Les ateliers interprofessionnels sont co-portés par J. Choplin, E. Lebreton et J. Lorilleux. E. Mercier collabore également aux deux expériences.

6. Il s'agit de structures associatives impliquées de longue date dans l'intervention auprès des personnes migrantes, et notamment dans l'enseignement du français à visée d'insertion sociale et professionnelle. Ces partenaires œuvrent en région Centre Val-de-Loire et en Normandie.

7. Le colloque «Les «francophones" devant les normes, 40 ans après Les Français devant la norme - L'(in)sécurité linguistique aujourd'hui : perspectives in(ter)disciplinaires », s'est déroulé à Tours du 13 au15 juin 2018.

8. Parmi ces professionnels figurent nos partenaires de stage pour les étudiants de Master FLE/S ainsi que des collègues ou des employeurs de doctorants de l'équipe qui sont salariés dans ces structures, dont certains sont venus accompagnés de leurs apprenants. Cette précision vise à témoigner de notre projet de travailler plus étroitement avec les professionnels qui participent à la formation de nos étudiants.

9. «traduction de la dimension dialogale avec autrui qui fait prendre conscience de notre altérité » Feussi, $2018: 185$.

10. Catégories délimitées par les statuts institutionnels.

\section{RÉSUMÉS}

Les travaux sur les recherches collaboratives en didactique des langues tendent à distinguer divers types de recherches en fonction notamment de l'implication des différents acteurs, des modalités de collaboration, et de la nature des connaissances construites. En déstabilisant les catégories habituellement mobilisées dans ce paradigme à l'appui de nos propres expériences de recherches "collaboratives", nous explorons les dimensions épistémologiques, éthiques et relationnelles qui fondent notre engagement dans ces démarches, afin d'envisager différemment, i.e. sous l'angle expérientiel, la manière dont les savoirs s'y construisent.

Die Arbeiten über die Kollaborativforschung im Bereich der Didaktik der Sprachen weisen darauf hin, gewisse Kategorien der Forschung zu unterscheiden, besonders im Hinblick auf den Einsatz der verschiedenen Akteure, auf die Formen der Mitarbeit und auf die Natur der aufgebauten Wissen. Auf Grund unserer eigenen Erfahrungen bei der Kollaborativforschung stellen wir die herkömmlich in diesem Paradigma mobilisierten Kategorien in Frage. In diesem Zusammenhang erforschen wir die epistemologischen, ethischen und relationellen Dimensionen, auf welchen unser Engagement in dieser Vorgehensweise beruht, damit wir die Wissenskonstruktion anders, nämlich erfahrungsgestützt, in Betracht ziehen können.

\section{INDEX}

Mots-clés : recherche collaborative, engagement, éthique, épistémologie, inconfort positif Keywords : collaborative research, involvement, ethics, epistemology, positive discomfort 


\section{AUTEURS}

\section{EMILIE LEBRETON}

LPL- UMR7309

Aix-Marseille Université

Emilie Lebreton est MCF en Didactique du FLE, ses recherches portent sur l'appropriation du français en contexte migratoire. Les recherches collaboratives menées avec les acteurs diversement impliqués auprès de personnes migrantes engagées en formation linguistique, la conduisent à développer des réflexions concernant : l'articulation langue(s) et insertion(s), la dimension langagière de la scolarisation et de la socialisation, les conceptions du métier d'enseignant/formateur de FLE/S.

emilie.lebreton@univ-amu.fr

\section{JOANNA LORILLEUX}

Dynadiv EA4428

Université de Tours

Joanna Lorilleux est MCF à l'université de Tours (SDL didactique des langues), ses recherches concernent les situations où le français contribue à la formation des personnes qui se l'approprient, qu'elles soient adultes ou enfants. Ancrées dans des approches qualitatives d'inspiration phénoménologique-herméneutique, ces recherches s'articulent autour des mots clés suivants : français langue seconde, (pluri)littératie, articulation langue(s) et insertion(s), appropriation, approches artistiques.

joanna.lorilleux@univ-tours.fr 